\title{
The effect of autologous leukocyte platelet rich fibrin on the rate of orthodontic tooth movement: A prospective randomized clinical trial
}

\author{
Azita Tehranchi ${ }^{1}$, Hossein Behnia ${ }^{2}$, Fereydoun Pourdanesh ${ }^{3}$, Parsa Behnia ${ }^{4}$, \\ Nelson Pinto ${ }^{5}$, Farnaz Younessian ${ }^{6}$
}

Correspondence: Dr. Parsa Behnia

Email: parsabehnia1@gmail.com

\begin{abstract}
'Dental Research Center, Dental Research Institute, Dental School, Shahid Beheshti University of Medical Sciences, Tehran, Iran,

2Dentofacial Deformities Research Center, Dental Research Institute, Dental School, Shahid Beheshti University of Medical Sciences, Tehran, Iran, ${ }^{3}$ Department of Oral and Maxillofacial Surgery, Dental School, Shahid Beheshti University of Medical Sciences, Tehran, Iran,

${ }^{4}$ Postgraduate Student, Department of Oral and Maxillofacial Surgery, Dental School, Shahid Beheshti University of Medical Sciences, Tehran, Iran, ${ }^{5}$ Department of Periodontic and Implant Dentistry, Faculty of Dentistry, University of The Andes, Las Condes, Santiago, Chile,

${ }^{6}$ Department of Orthodontics, College of Dental Medicine, Nova Southeastern University, Fort Lauderdale, FL 33328, USA
\end{abstract}

\section{ABSTRACT}

Objective: The aim of this study was to evaluate the effect of LPRF, placed in extraction sockets, on orthodontic tooth movement (OTM). Materials and Methods: Thirty extraction sockets from eight patients (five males, three females, with a mean age of 17.37 years; range 12-25 years) requiring extraction of first premolars based on their orthodontic treatment plan participated in this split-mouth clinical trial. In one randomly selected quadrant of each jaw, the extraction socket was preserved as the experimental group by immediate placement of LPRF in the extraction socket. The other quadrant served as the control group for secondary healing. Immediately, the teeth adjacent to the defects were pulled together by a NiTi closed-coil spring with constant force. A piece of $0.016 \times 0.022$-inch stainless steel wire was used as the main arch wire. The amount of OTM was measured on the study casts at eight time points with 2-week intervals for 3 months. Analysis of random effect model was performed for the purpose of comparison between the experimental and control groups. Results: According to the random effect model, a statistically significant difference was found between the experimental and control group in rate of OTM $(P=0.006)$.Conclusion: According to the results, application of LPRF, as an interdisciplinary approach combining orthodontics and surgery, may accelerate OTM, particularly in extraction cases.

This is an open access journal, and articles are distributed under the terms of the Creative Commons Attribution-NonCommercial-ShareAlike 4.0 License, which allows others to remix, tweak, and build upon the work non-commercially, as long as appropriate credit is given and the new creations are licensed under the identical terms.

\begin{tabular}{|l|l|}
\hline \multicolumn{2}{|c|}{ Access this article online } \\
\hline Quick Response Code: \\
\hline
\end{tabular}

For reprints contact: reprints@medknow.com

How to cite this article: Tehranchi $A$, Behnia $H$, Pourdanesh $F$ Behnia P, Pinto N, Younessian F. The effect of autologous leukocyte platelet rich fibrin on the rate of orthodontic tooth movement: $A$ prospective randomized clinical trial. Eur J Dent 2018;12:350-7.

DOI: 10.4103/ejd.ejd_424_17 
Key words: Leukocyte platelet rich fibrin, orthodontic tooth movement, plasma, platelet concentrate, platelet-rich fibrin, platelet-rich plasma

\section{INTRODUCTION}

To date, several methods have been applied to enhance the postoperative healing process and decrease inflammation. ${ }^{[1-3]}$ Considering the local release of growth factors, platelets can be used as an adjunct to stimulate the regenerative capacity of periosteum and enhance bone healing, especially in postextraction cases. ${ }^{[4-7]}$ Blood vessels provide the necessary growth factors and inhibitors to initiate the osteogenic biomineralization cascade. Inevitable injury to blood vessels during oral surgical procedures causes blood extravasation and subsequent platelet aggregation and fibrin clot formation. Platelet activation results in formation of blood clots and platelet plugs and subsequent secretion of bioactive proteins, necessary for tissue regeneration and repair. ${ }^{[2,6,8]}$

Considering the bioactive properties of platelets in the healing process, they can be used as a valuable therapeutic adjunct in medicine and dentistry. ${ }^{[9,10]}$ Platelet-rich plasma (PRP) and leukocyte platelet-rich fibrin (LPRF) are the two main autologous products derived from platelets. ${ }^{[11-15]}$ Many studies have investigated the potential biological differences between these two platelet concentrates. ${ }^{[15,16]}$ Although they are both clinically effective in accelerating the healing process, platelet-rich fibrin (PRF) is low cost and easy to use and may be added to other bone substitutes. ${ }^{[17]}$ Compared to PRP, LPRF and PRF have demonstrated more sustained release of growth factors from the fine and flexible fibrin matrix in their structure. ${ }^{[15,18]}$ Furthermore, the presence of high concentrations of leukocytes in LPRF compared to PRF plays a significant role in enhanced release of some crucial growth factors such as transforming growth factor-beta $1 .{ }^{[1]}$

Resorptive remodeling of the alveolar ridge commonly occurs following tooth extraction. This process may be beneficial in fixed orthodontic treatment of patients with severe crowding. ${ }^{[20-22]}$ Literature is rich with studies on the application of various bioactive grafts to increase the bone maturation rate and enhance the rate of orthodontic tooth movement (OTM). ${ }^{[23]}$ Adequate volume of alveolar bone is a prerequisite for successful OTM during space closure. However, application of different graft materials may enhance the bone filling process and consequently shorten the "regional acceleratory phenomenon" cascade, which includes the release of various growth factors after tooth extraction. LPRF contains concentrated growth factors such as platelet-derived growth factor, transforming growth factor-beta, and insulin-like growth factor 1 , which enriches the blood clot formed in the extraction socket and subsequently enhances wound healing and bone regeneration with no inhibitory effect on the natural healing process. ${ }^{[19,20,23]}$

Regarding the acceleration of tooth movement, limited publications are available on the efficacy of submucosal injection of PRP to accelerate OTM and preserve the bone. ${ }^{[24-26]}$ Considering the simultaneous positive effect of PRP/LPRF on bone healing, socket preservation, and acceleration of tooth movement, the current study tested the application of LPRF in tooth extraction sockets to evaluate its efficacy for acceleration of space closure phase of orthodontic treatment. This study sought to evaluate whether LPRF application can accelerate OTM. To the best of our knowledge, this is the first study on the efficacy of LPRF for acceleration of OTM.

\section{MATERIALS AND METHODS}

\section{Study design}

This split-mouth clinical trial evaluated the efficacy of application of LPRF in extraction sockets for acceleration of OTM in fixed orthodontic patients. A split-mouth design was used to limit the effect of interpersonal variations on response to LPRF. The study was conducted in the Department of Orthodontics and Dentofacial Orthopedics of Shahid Beheshti University of Medical Sciences between March 2015 and March 2016. This clinical trial was registered in www.irct.ir (IRCT2015100724405N1) and followed the CONSORT statement as a guide for study design. Ethical approval was also obtained from the Ethics Committee of Dental Research Center at Shahid Beheshti University of Medical Sciences (protocol approval number: 9310).

\section{Sample size calculation}

Sample size was calculated to be thirty extraction sockets ( $n=15$ in each group) assuming the mean difference and standard deviation (SD) of 0.95 , type 1 error $(\alpha)$ of 0.05 and type 2 error $(\beta)$ of 0.1 to achieve a statistical power of $90 \%$ using $N=(\mathrm{Z} 1-\alpha / 2+\mathrm{Z} 1-\beta)^{2}$ $\times \sigma^{2} /(\Delta \mu)^{2}$ with assumptions of $\alpha$ (significance level $)=$ $0.05 \geq Z 1-\alpha / 2=1.96, \beta=0.1$ (statistical power $=0.9$ ) $\geq Z 1-\beta=1.28, \sigma=0.95, \Delta \mu=0.95$. 


\section{Participants and randomization}

Participants were selected from patients referred to the Department of Orthodontics and Dentofacial Orthopedics at Shahid Beheshti University of Medical Sciences. Male and female orthodontic patients 12 years and older with extraction treatment plan who met the following inclusion criteria were included presence of all maxillary and mandibular permanent teeth except for third molars, comprehensive orthodontic treatment plan of bilateral symmetric extraction of premolar teeth, no medication intake or systemic disease, and full banding/bonding of teeth in both arches. The exclusion criteria were pregnancy, history of previous orthodontic treatment, nonextraction treatment plan, syndromic patients, systemic diseases, or medication intake such as nonsteroidal anti-inflammatory drugs, which would interfere with OTM and history of previous orthodontic treatment. Each patient signed an informed consent form after receiving a thorough explanation regarding the study. In each jaw, the LPRF application quadrant was chosen by drawing lot, done by the corresponding author. In one-quadrant of each jaw, the extraction socket was preserved by immediate placement of LPRF as the experimental group, while the other side served as the control group for secondary healing.

\section{Preparation of leukocyte platelet-rich fibrin}

The protocol for LPRF preparation was simple and included the collection of whole venous blood from the brachial vein using a $10 \mathrm{~mL}$ syringe. The collected blood was transferred into two sterile vacutainer tubes $(9 \mathrm{~mL})$ without anticoagulant and were placed symmetrically into the centrifuge device. The intraspin tubes were immediately centrifuged ( $\mathrm{PCO}_{2}$ Process) at $2700 \mathrm{rpm}$ for $12 \mathrm{~min}$, after which, three layers were formed: red blood cells at the bottom, upper straw-colored cellular plasma, and the middle fraction containing the fibrin clot and platelets. The upper straw-colored layer was discarded, and the middle fraction was collected, $2 \mathrm{~mm}$ below the lower dividing line, which was LPRF. Fibrinogen which is initially concentrated in the upper part of the tube is combined with the circulating thrombin following centrifugation to form fibrin. A fibrin clot is then formed in the middle of the tube, right between the red corpuscles at the bottom and acellular plasma at the top. Platelets are trapped massively in the fibrin mesh [Figure 1a and b].

The central part includes platelets trapped massively in the fibrin mesh. The success of this technique entirely depends on the time interval between blood collection and its centrifugation, which should be minimized. The blood sample without anticoagulant starts to coagulate almost immediately upon contact with the glass and decreases the required centrifugation time to concentrate fibrinogen. Adherence to the correct preparation protocol and quick handling are critical to obtain clinically usable LPRF clot charged with serum and platelets. Resistant autologous fibrin membranes may be available by driving out the fluids trapped in the fibrin matrix.

\section{Orthodontic tooth movement}

Within the scope of orthodontic treatment with fixed appliances (ROTH American Orthodontics Master/Mini-Series 0.022-inch slot), patients requiring extraction of their first premolars according to their orthodontic treatment plan after the leveling phase of treatment underwent augmentation of extraction socket with LPRF unilaterally immediately after extraction. Extraction of first premolars was performed a traumatically on both sides. On one side of each jaw, the extraction socket was preserved by immediate placement of LPRF in the extraction socket as the experimental group and the other sides served as the control for secondary healing. The LPRF plugs were placed gently into the socket, and the sockets were sutured using 4-0 Vicryl sutures (Ethicon). The teeth adjacent to the defect were then pulled together by a NiTi closed-coil spring (Ormco ${ }^{\circledR}$, Orange, California, USA) with constant level of force. A piece of $0.016 \times 0.022$-inch stainless steel wire was used as the main archwire. The sites were examined weekly for any appliance dislodgment. The treatment was considered finished when the amount of OTM was considered clinically enough based on the clinical appraisal of individual pretreatment crowding and proposed treatment plan.

The amount of OTM was measured by comparing the change in horizontal linear distance between the mid-marginal ridges of the adjacent teeth on a regular

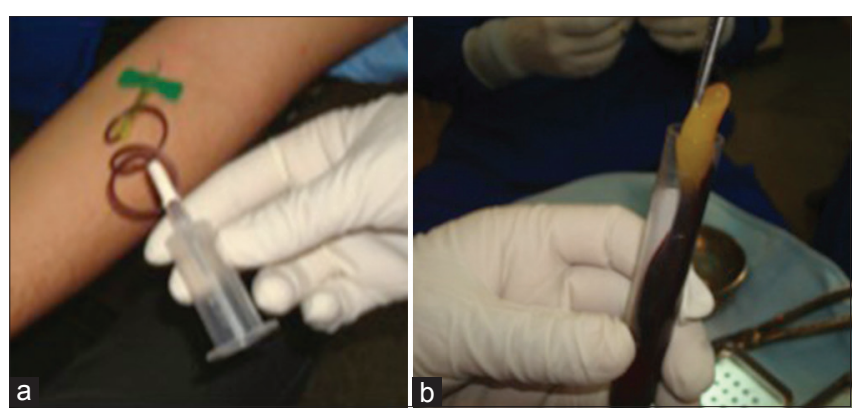

Figure 1: (a) Collection of venous blood from the brachial vein using a $10 \mathrm{~mL}$ syringe. (b) Platelets are trapped massively in the fibrin mesh 
basis every 2 weeks for 4 months (eight time points: before placement of LPRF [T0] and 2 weeks [T1], 4 weeks [T2], 6 weeks [T3], 8 weeks [T4], 10 weeks [T5], 12 weeks [T6], 14 weeks [T7], and 16 weeks [T8]) after the study commencement until adequate canine retraction was achieved which is different in each socket [Figure 2a and $b$ ]. If this distance decreases more in experimental side, it means that the teeth moved faster than control side.

\section{Statistical analysis}

Statistical analysis was performed using SPSS 18 (SPSS Inc., PASW statistics for windows, Version 18.1. ChicGO). In this split-mouth clinical trial, descriptive statistics, including the mean and SD of OTM in each group, were measured at eight different time points. The independent sample $t$-test was used to evaluate the equality of means in both experimental and control group. The random effect model was used for the comparison between the experimental and control groups, and variables, which could be considered as personal differences, were entered into the model using a split mouth design.

\section{RESULTS}

Thirty extraction sockets of eight patients (five males, three females, mean age of 17.37 years

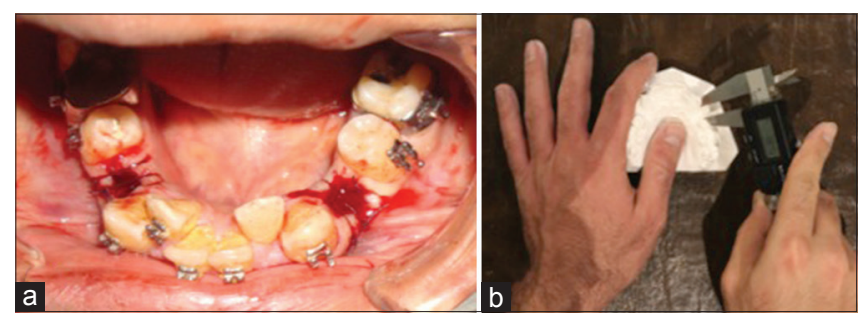

Figure 2: (a) The extraction socket was preserved by immediate placement of LPRF in the extraction socket as the experimental group and the other side served as the control group for secondary healing. (b) The exact amount of tooth movement were measured on the study casts using a digital caliper and SD of 12.48 years, and range 12-25 years) were assessed in this study. All patients completed the follow-up period. In seven patients, both maxillary and mandibular arches and in one patient only the mandibular arch were assessed. Table 1 shows the mean and SD of tooth movement in the control and experimental groups.

In all eight time points, the mean linear measurements between mid-marginal ridges of teeth adjacent to extraction sites were less in experimental groups compared to control. This distance decreased more in experimental side which means that the teeth moved faster than control side.

According to the random effect model, the experimental group (LPRF placed in the extraction socket) showed higher rate of OTM $(P=0.006)$. Figure 3 shows the pattern of tooth movement in the two groups. The graph shows the higher rate of OTM in the experimental group $(P=0.006)$. According to the random effect model, the teeth in

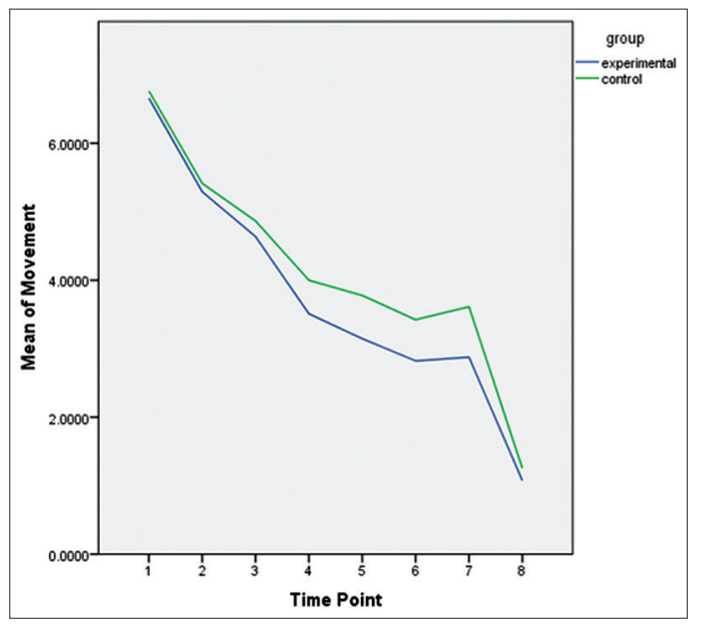

Figure 3: Amount the distance between marginal ridges of the teeth adjacent to sockets in millimeters at different time points. Data represent the mean values in the experimental and control groups

\begin{tabular}{|c|c|c|c|c|c|c|c|c|c|c|}
\hline Time & Point & Experimental & Control & $95 \%$ & $\mathrm{Cl}$ & $n$ & Mean & SD & $95 \%$ & $\mathrm{Cl}$ \\
\hline 1 & 15 & 6.65 & 0.834 & 6.19 & 7.11 & 15 & 6.762 & 0.763 & 6.33 & 7.18 \\
\hline 2 & 15 & 5.290 & 1.783 & 4.30 & 6.27 & 15 & 5.412 & 1.738 & 4.44 & 6.37 \\
\hline 3 & 15 & 4.636 & 1.824 & 3.62 & 5.64 & 15 & 4.865 & 1.764 & 3.88 & 5.84 \\
\hline 4 & 15 & 3.510 & 2.023 & 2.38 & 4.63 & 15 & 3.998 & 2.138 & 2.81 & 5.18 \\
\hline 5 & 14 & 3.145 & 1.617 & 2.21 & 4.07 & 14 & 3.777 & 1.923 & 2.66 & 4.88 \\
\hline 6 & 13 & 2.822 & 1.337 & 2.01 & 3.63 & 13 & 3.423 & 1.742 & 2.37 & 4.47 \\
\hline 7 & 9 & 2.875 & 0.499 & 2.49 & 3.25 & 9 & 3.612 & 1.375 & 2.55 & 4.66 \\
\hline 8 & 4 & 1.075 & 0.153 & 0.83 & 1.31 & 4 & 1.255 & 0.100 & 1.09 & 1.41 \\
\hline Total & 100 & 4.123 & 2.081 & -0.86 & 0.80 & 100 & 4.505 & 2.070 & -0.71 & 0.94 \\
\hline
\end{tabular}




\begin{tabular}{|c|c|c|c|c|}
\hline Group & Arch & Mean & SD & $P$ \\
\hline \multirow[t]{3}{*}{ Experimental } & Upper (7) & 6.76 & 0.538 & 0.94 \\
\hline & Lower (8) & 6.56 & 1.058 & \\
\hline & Total (15) & 6.65 & 0.834 & \\
\hline \multirow[t]{3}{*}{ Control } & Upper (7) & 6.96 & 0.695 & 0.77 \\
\hline & Lower (8) & 6.58 & 0.819 & \\
\hline & Total (15) & 6.76 & 0.763 & \\
\hline \multirow[t]{2}{*}{ Total } & Upper (14) & 6.86 & 0.606 & 0.626 \\
\hline & Lower (16) & 6.57 & 0.914 & \\
\hline
\end{tabular}

the maxillary compared to mandibular arches in both the experimental and control group did not show any significant difference in the rate of OTM $[P=0.9$ and $P=0.77$, respectively, Table 2].

Table 2 shows the mean and SD of tooth movement (millimeters) in both the control and experimental groups in the maxillary and mandibular arches.

\section{DISCUSSION}

The results of this study demonstrated that the distance between mid-marginal ridge points of crowns adjacent to extraction sites was less in experimental groups; therefore, it showed the possible positive efficacy of LPRF application in the extraction socket for acceleration of OTM including anchorage loss of posterior teeth. It means that anterior and posterior teeth adjacent to extraction sites moved faster toward each other in experimental groups. To the best of our knowledge, this is the first pilot study aiming to evaluate the efficacy of LPRF for acceleration of OTM in humans. To date, many surgically assisted approaches such as periodontally accelerated osteogenic orthodontics, corticotomy, and micro-osteoperforation with and without using PRP/LPRF have been developed to decrease the treatment time of fixed orthodontic patients. These approaches are all based on the regional acceleratory phenomenon. ${ }^{[27-31]}$ Researchers tried to shift the invasive surgically assisted techniques to more conservative approaches including micro-osteoperforation, ${ }^{[31]}$ piezopuncture, ${ }^{[32]}$ and very recently mini-implant-facilitated micro-osteoperforation, ${ }^{[33]}$ which eliminate the need for flaps, bone grafting, and suturing. However, these techniques may still traumatize the surrounding bone, undermine the periodontal support of teeth, and cause patient discomfort. ${ }^{[33]}$ In addition, the intensity and extensity of their acceleratory effect depend on the intensity and extensity of the surgical insult, and therefore, conservative approaches might not be able to trigger a distinctive acceleratory effect on OTM. ${ }^{[34]}$ To simulate the effects of surgical insult without actual surgical trauma, local injection of growth factors and cytokines, and recently, autologous PRP were introduced to stimulate alveolar bone remodeling. ${ }^{[25,26,35]}$

PRP was first introduced to dentistry in 1998 to be combined with autogenous bone grafts to expedite bone maturation and result in higher bone density. ${ }^{[36]}$ Hoaglin and Lines also conducted a study to evaluate the use of PRF for prevention of localized osteitis following lower third molar extraction. ${ }^{[6]}$ This retrospective review demonstrated that preventative treatment of localized osteitis could be accomplished using a low cost, autogenous, soluble, biologic material, PRF, and that PRF enhanced third molar socket healing/clot retention and greatly decreased the clinical time required for postoperative management of localized osteitis. ${ }^{[6]}$ Since then, many studies have evaluated the use of LPRF and PRF to facilitate implant placement and periapical surgeries, ${ }^{[37,38]}$ revascularization procedures, ${ }^{[39]}$ perforation repair, and also bone regeneration in oral and maxillofacial region. ${ }^{[38]}$

Considering the efficiency of PRP for healing of the alveolar socket after tooth extraction, there are controversies regarding its effect on postoperative pain, swelling, bleeding, and postoperative discomfort. ${ }^{[13,40,41]}$ Radiographic examinations revealed an early and significant increased radiographic density at the PRP-treated sockets in comparison to the ipsilateral not PRP treated sites, ${ }^{[42,43]}$ demonstrating the effect of PRP on early phase of bone healing. However, in a prospective split-mouth study conducted by Arenaz-Bua et al., this acceleration in bone formation was not reported to last for more than 6 months. ${ }^{[41]}$

On the other hand, considering the inhibitory effect of high concentration of PRP on bone cell division and bone density, a submucosal injection of PRP for acceleration of OTM was reported by Liou in 2016. ${ }^{[24]}$ This study reported dose-dependent application of PRP for acceleration of tooth movement and for alveolar bone loss at the pressure side of en masse anterior retraction. However, submucosal injection of PRP is superior to other surgically assisted methods since it is a noninvasive and clinically feasible method. ${ }^{[24]}$ In this technique, PRP is injected through the attached gingiva into the oral mucosa. The 
disadvantages of this technique include the necessity of administration of local anesthesia before injection for pain control, probability of PRP leakage during injection, and also the possibility of postinjection pain, discomfort, and mucosal swelling. In this study, $15 \%$ of patients reported severe postinjection pain, which was correlated with the PRP concentration level. ${ }^{[24]}$ On the other hand, in orthodontic patients with extraction treatment plan, LPRF/PRP can be placed in the extraction sockets and there would be no need for its submucosal injection. However, controversies exist regarding the potential benefit of each procedure and further studies are needed to fully clarify the advantages of each method of delivery in extraction patients.

In vitro and in vivo studies have shown that the release of growth and healing factors peaks at around 7 days after buccal vestibular mucosal injection in rat models. However, this acceleratory effect was transient and seemed to decrease over the next $2-3$ weeks. ${ }^{[15,25]}$ In a study by Güleç et al., on rat models, the difference in the amount of tooth movement between the experimental (high concentration PRP) and control group was significant at 7,14 , and 21 days. This result was in accordance with our study, in which the total amount of tooth movement in the experimental group was significantly higher at T1 ( 2 weeks $=14$ days) and T2 ( 4 weeks $=28$ days). However, in the study by Güleç et al., the study duration was limited to 21 days after buccal vestibular injection, and long-term effects were not evaluated in their study. ${ }^{[25]}$

In a study by Liou on humans, it was stated that the clinical effect of a single submucosal injection of PRP could last for 5-6 months clinically. The fastest rate of acceleration was reported to be during the $2^{\text {nd }}-4^{\text {th }}$ month after the injection. ${ }^{[24]}$ This result was in accordance with our study, in which the total amount of tooth movement in the experimental group was significantly higher at $\mathrm{T} 1$ ( 2 weeks $=14$ days $)$ and T2 ( 4 weeks $=28$ days $)$. In addition, based on this transient effect, they suggested a multi-PRP injection protocol based on the clinical objective including a single injection of PRP at the beginning of treatment for the purpose of alignment and leveling and another booster injection at 6 months after the first injection for the purpose of anterior retraction or posterior protraction. ${ }^{[24]}$

One limitation of this study was lack of precise evaluation of whole blood and LPRF content and concentration of growth factors in each patient. Liou assessed the platelet count in $1 \mathrm{~mL}$ of the collected blood sample and final PRP sample. ${ }^{[24]}$ In a recent study by Güleç et al., in 2017, the rate of OTM was evaluated in rats histomorphometrically after submucosal injection of high and moderate concentrations of PRP. ${ }^{[25]}$ They demonstrated that both high and moderate concentrations of PRP had a transient acceleratory effect on OTM in rats. This result was in contrast to the previous studies that reported the inhibitory effect of PRP on bone metabolism only in high concentrations of PRP. ${ }^{[44]}$ The authors discussed that the reason may be the presence of mechanical force (dynamic loading) in contrast to static loading in the reported study. ${ }^{[25]}$ Since PRP has a concentration-dependent effect on bone turnover ${ }^{[4]}$ and also OTM, ${ }^{[25]}$ it may be stated that different LPRF concentrations may also have different effects on bone turnover and OTM. ${ }^{[25]}$ However, the split-mouth design of this study aimed to compensate for these possible interpersonal variations. Nevertheless, for future studies, it is highly recommended to determine the concentrations of platelets and leucocytes in the whole blood and LPRF samples by the use of ELISA before their application.

LPRF, similar to PRP products, is a mixture of various growth factors, cytokines, and enzymes, which may have overlapping biological effects and the exact mechanism of action of each ingredient is still unclear. ${ }^{[45]}$ Many of these elements might demonstrate anti-inflammatory effects, responsible for the increased tissue healing capacity, and at the same time, many cytokines such as tissue necrosis factors might aggravate the inflammatory response and lead to accelerated tooth movement. ${ }^{[46]}$ As stated in PRP studies, LPRF can also promote both inflammatory and anti-inflammatory responses and their exact effect could be mainly related to the timing of release, concentration, and content of its growth factors. ${ }^{[4]}$

Considering the lack of available evidence on the effect of LPRF on the rate of tooth movement in humans, our result is not comparable to the findings of other studies in this field. The major limitation of this study was limited sample size and the relatively negligible observed clinical effect on acceleration of OTM.

A major strength of this study was that it showed the possibility of application of autologous blood-derived LPRF in humans compared to allogenic PRP application in rat models. Therefore, the observed result could not be attributed to immune reaction to the allogenic PRP injection. ${ }^{[25,45]}$ However, the inconvenience related to the need for venipuncture 
and blood-drawing procedure for preparation of LPRF should be considered before clinical application of this method. Furthermore, more randomized clinical trials are recommended.

\section{CONCLUSION}

More decrease in horizontal linear measurement between the mid-marginal ridges of teeth in experimental groups than control groups means that application of LPRF may accelerate OTM, particularly in cases with extraction treatment plan.

\section{Acknowledgement}

This study was extracted from a undergraduate thesis of Parsa Behnia under supervision of Dr. Fereydoun Pourdanesh at Shahid Beheshti School of Dentistry. The authors wish to thank the Dental Research Center, Shahid Beheshti University of Medical Sciences, for support of this project.

\section{Financial support and sponsorship}

Nil.

\section{Conflicts of interest}

There are no conflicts of interest.

\section{REFERENCES}

1. Singh A, Kohli M, Gupta N. Platelet rich fibrin: A novel approach for osseous regeneration. J Maxillofac Oral Surg 2012;11:430-4.

2. Hatakeyama I, Marukawa E, Takahashi Y, Omura K. Effects of platelet-poor plasma, platelet-rich plasma, and platelet-rich fibrin on healing of extraction sockets with buccal dehiscence in dogs. Tissue Eng Part A 2014;20:874-82.

3. Marrelli M, Tatullo M. Influence of PRF in the healing of bone and gingival tissues. Clinical and histological evaluations. Eur Rev Med Pharmacol Sci 2013;17:1958-62.

4. Schliephake H. Clinical efficacy of growth factors to enhance tissue repair in oral and maxillofacial reconstruction: A systematic review. Clin Implant Dent Relat Res 2015;17:247-73.

5. Suttapreyasri S, Leepong N. Influence of platelet-rich fibrin on alveolar ridge preservation. J Craniofac Surg 2013;24:1088-94.

6. Hoaglin DR, Lines GK. Prevention of localized osteitis in mandibular third-molar sites using platelet-rich fibrin. Int J Dent 2013;2013:875380.

7. Del Fabbro M, Corbella S, Taschieri S, Francetti L, Weinstein R. Autologous platelet concentrate for post-extraction socket healing: A systematic review. Eur J Oral Implantol 2014;7:333-44.

8. Del Corso M, Mazor Z, Rutkowski JL, Dohan Ehrenfest DM. The use of leukocyte- and platelet-rich fibrin during immediate postextractive implantation and loading for the esthetic replacement of a fractured maxillary central incisor. J Oral Implantol 2012;38:181-7.

9. Chang YC, Zhao JH. Effects of platelet-rich fibrin on human periodontal ligament fibroblasts and application for periodontal infrabony defects. Aust Dent J 2011;56:365-71.

10. Sammartino G, Dohan Ehrenfest DM, Carile F, Tia M, Bucci P. Prevention of hemorrhagic complications after dental extractions into open heart surgery patients under anticoagulant therapy: The use of leukocyte- and platelet-rich fibrin. J Oral Implantol 2011;37:681-90.

11. Taschieri S, Del Fabbro M. Postextraction osteotome sinus floor elevation technique using plasma-rich growth factors. Implant Dent 2011;20:418-24.

12. Ruga E, Gallesio C, Boffano P. Platelet-rich fibrin and piezoelectric surgery: A safe technique for the prevention of periodontal complications in third molar surgery. J Craniofac Surg 2011;22:1951-5.

13. Del Corso M, Vervelle A, Simonpieri A, Jimbo R, Inchingolo $F$ Sammartino G, et al. Current knowledge and perspectives for the use of platelet-rich plasma (PRP) and platelet-rich fibrin (PRF) in oral and maxillofacial surgery part 1: Periodontal and dentoalveolar surgery. Curr Pharm Biotechnol 2012;13:1207-30.

14. Simonpieri A, Del Corso M, Vervelle A, Jimbo R, Inchingolo F, Sammartino G, et al. Current knowledge and perspectives for the use of platelet-rich plasma (PRP) and platelet-rich fibrin (PRF) in oral and maxillofacial surgery part 2: Bone graft, implant and reconstructive surgery. Curr Pharm Biotechnol 2012;13:1231-56.

15. Schär MO, Diaz-Romero J, Kohl S, Zumstein MA, Nesic D. Platelet-rich concentrates differentially release growth factors and induce cell migration in vitro. Clin Orthop Relat Res 2015;473:1635-43.

16. Simon BI, Gupta P, Tajbakhsh S. Quantitative evaluation of extraction socket healing following the use of autologous platelet-rich fibrin matrix in humans. Int J Periodontics Restorative Dent 2011;31:285-95.

17. Baeyens W, Glineur R, Evrard L. The use of platelet concentrates: Platelet-rich plasma (PRP) and platelet-rich fibrin (PRF) in bone reconstruction prior to dental implant surgery. Rev Med Brux 2010;31:521-7.

18. Peck MT, Marnewick J, Stephen L. Alveolar ridge preservation using leukocyte and platelet-rich fibrin: A report of a case. Case Rep Dent 2011;2011:345048.

19. Huang FM, Yang SF, Zhao JH, Chang YC. Platelet-rich fibrin increases proliferation and differentiation of human dental pulp cells. J Endod 2010;36:1628-32.

20. Yuan X, Luo S, Shen G. Experimental study on selecting optimal time of orthodontic tooth movement into extraction sites. Hua Xi Kou Qiang Yi Xue Za Zhi 2003;21:311-3.

21. Kocyigit ID, Tuz HH, Alp YE, Atil F, Tekin U, Coskunses FM, et al. Correction of postsurgical alveolar ridge defect with vertical alveolar distraction of the onlay block graft. J Craniofac Surg 2012;23:1550-2.

22. Gürbüzer B, Pikdöken L, Tunali M, Urhan M, Küçükodaci Z, Ercan F, et al. Scintigraphic evaluation of osteoblastic activity in extraction sockets treated with platelet-rich fibrin. J Oral Maxillofac Surg 2010;68:980-9.

23. Dohan Ehrenfest DM, Bielecki T, Jimbo R, Barbé G, Del Corso M, Inchingolo $\mathrm{F}$, et al. Do the fibrin architecture and leukocyte content influence the growth factor release of platelet concentrates? An evidence-based answer comparing a pure platelet-rich plasma (P-PRP) gel and a leukocyte- and platelet-rich fibrin (L-PRF). Curr Pharm Biotechnol 2012;13:1145-52.

24. Liou EJ. The development of submucosal injection of platelet rich plasma for accelerating orthodontic tooth movement and preserving pressure side alveolar bone. APOS Trends Orthod 2016;6:5.

25. Güleç A, Bakkalbaşı BÇ, Cumbul A, Uslu Ü, Alev B, Yarat A, et al. Effects of local platelet-rich plasma injection on the rate of orthodontic tooth movement in a rat model: A histomorphometric study. Am J Orthod Dentofacial Orthop 2017;151:92-104.

26. Rashid A, ElSharaby FA, Nassef EM, Mehanni S, Mostafa YA. Effect of platelet-rich plasma on orthodontic tooth movement in dogs. Orthod Craniofac Res 2017;20:102-10.

27. Munoz F, Jiménez C, Espinoza D, Vervelle A, Beugnet J, Haidar Z, et al. Use of leukocyte and platelet-rich fibrin (L-PRF) in periodontally accelerated osteogenic orthodontics (PAOO): Clinical effects on edema and pain. J Clin Exp Dent 2016;8:e119-24.

28. Alfawal AM, Hajeer MY, Ajaj MA, Hamadah O, Brad B. Effectiveness of minimally invasive surgical procedures in the acceleration of tooth movement: A systematic review and meta-analysis. Prog Orthod 2016;17:33.

29. Campbell JH. Periodontally accelerated osteogenic orthodontics. J Oral Maxillofac Surg 2017;75:6.

30. Tsai CY, Yang TK, Hsieh HY, Yang LY. Comparison of the effects of micro-osteoperforation and corticision on the rate of orthodontic tooth movement in rats. Angle Orthod 2016;86:558-64.

31. Alikhani M, Raptis M, Zoldan B, Sangsuwon C, Lee YB, Alyami B, et al. Effect of micro-osteoperforations on the rate of tooth movement. Am J Orthod Dentofacial Orthop 2013;144:639-48.

32. Hoffmann S, Papadopoulos N, Visel D, Visel T, Jost-Brinkmann PG, Präger TM, et al. Influence of piezotomy and osteoperforation of the alveolar process on the rate of orthodontic tooth movement: A systematic review. J Orofac Orthop 2017;78:301-11. 
33. Cheung T, Park J, Lee D, Kim C, Olson J, Javadi S, et al. Ability of mini-implant-facilitated micro-osteoperforations to accelerate tooth movement in rats. Am J Orthod Dentofacial Orthop 2016;150:958-67.

34. McBride MD, Campbell PM, Opperman LA, Dechow PC, Buschang PH. How does the amount of surgical insult affect bone around moving teeth? Am J Orthod Dentofacial Orthop 2014;145:S92-9.

35. Simon D, Manuel S, Geetha V, Naik BR. Potential for osseous regeneration of platelet-rich plasma - A comparative study in mandibular third molar sockets. Indian J Dent Res 2004;15:133-6.

36. Marx RE, Carlson ER, Eichstaedt RM, Schimmele SR, Strauss JE, Georgeff KR, et al. Platelet-rich plasma: Growth factor enhancement for bone grafts. Oral Surg Oral Med Oral Pathol Oral Radiol Endod 1998;85:638-46.

37. Rebentish PD, Umashetty G, Kaur H, Doizode T, Kaslekar M, Chowdhury $S$, et al. Platelet-rich fibrin: A boon in regenerative endodontics. Minerva Stomatol 2016;65:385-92.

38. Varghese MP, Manuel S, Kumar LK. Potential for osseous regeneration of platelet-rich fibrin-A comparative study in mandibular third molar impaction sockets. J Oral Maxillofac Surg 2017;75:1322-9.

39. Alagl A, Bedi S, Hassan K, AlHumaid J. Use of platelet-rich plasma for regeneration in non-vital immature permanent teeth: Clinical and cone-beam computed tomography evaluation. J Int Med Res 2017;45:583-93.

40. Albanese A, Licata ME, Polizzi B, Campisi G. Platelet-rich plasma (PRP) in dental and oral surgery: From the wound healing to bone regeneration. Immun Ageing 2013;10:23.

41. Arenaz-Búa J, Luaces-Rey R, Sironvalle-Soliva S, Otero-Rico A, Charro-Huerga E, Patiño-Seijas B, et al. A comparative study of platelet-rich plasma, hydroxyapatite, demineralized bone matrix and autologous bone to promote bone regeneration after mandibular impacted third molar extraction. Med Oral Patol Oral Cir Bucal 2010;15:e483-9.

42. Rutkowski JL, Johnson DA, Radio NM, Fennell JW. Platelet rich plasma to facilitate wound healing following tooth extraction. J Oral Implantol 2010;36:11-23.

43. Célio-Mariano R, de Melo WM, Carneiro-Avelino C. Comparative radiographic evaluation of alveolar bone healing associated with autologous platelet-rich plasma after impacted mandibular third molar surgery. J Oral Maxillofac Surg 2012;70:19-24.

44. Weibrich G, Hansen T, Kleis W, Buch R, Hitzler WE. Effect of platelet concentration in platelet-rich plasma on peri-implant bone regeneration. Bone 2004;34:665-71.

45. Marx RE. Platelet-rich plasma: Evidence to support its use. J Oral Maxillofac Surg 2004;62:489-96.

46. El-Sharkawy H, Kantarci A, Deady J, Hasturk H, Liu H, Alshahat M, et al. Platelet-rich plasma: Growth factors and pro- and anti-inflammatory properties. J Periodontol 2007;78:661-9.

47. Andia I, Maffulli N. Platelet-rich plasma for managing pain and inflammation in osteoarthritis. Nat Rev Rheumatol 2013;9:721-30. 